\title{
Familial Hemophagocytic Lymphohistiocytosis Type 3: Early Disease Onset and Unusual Manifestation in Sibling Cases
}

\author{
Junbin Huang ${ }^{1}$, Li Jiang ${ }^{2}$, Xiaojun $\mathrm{Wu}^{2}$, Yucai Cheng ${ }^{1}$, Chun Chen ${ }^{1}$ and Hongman Xue ${ }^{1,{ }^{*}}$ \\ ${ }^{1}$ Department of Pediatrics, The Seventh Affiliated Hospital, Sun Yat-Sen University, Shenzhen, China \\ ${ }^{2}$ Department of Pediatrics, Sun Yat-Sen Memorial Hospital, Sun Yat-Sen University, Guangzhou, China \\ "Corresponding author: Department of Pediatrics, The Seventh Affiliated Hospital, Sun Yat-Sen University, Shenzhen, China. Email: hongmanxue@126.com
}

Received 2018 July 14; Revised 2018 December 20; Accepted 2019 January 03.

Keywords: Familial Hemophagocytic Lymphohistiocytosis Type 3 (FHL-3), Compound Heterozygous, UNC13D, Disease Onset

\section{Dear Editor,}

Hemophagocytic lymphohistiocytosis (HLH) is a rare, life-threatening disease characterized by a heterogeneous spectrum of various causes, which leads to uncontrollable cytokine storm, and activation of lymphocytes and macrophages (1). Munc13 - 4 is a crucial effecter of the exocytosis of cytotoxic granules priming cytotoxic granule fusion, and UNC13D gene mutations result in defective cellular cytotoxicity and FHL type 3 (FHL3) disease onset (2).

Our case 1 was the first live-born child of a nonconsanguineous couple. The boy weighed $2.8 \mathrm{~kg}$, and was admitted to our neonatal intensive care unit (NICU) on 15 June 2014, immediately after birth at full term, which was by cesarean section because of pyrexia in the mother and abnormal fetal ultrasound findings. Physical examination on NICU admission showed pyrexia, tachypnea, and decreased breath sounds on the right side, as well as hepatomegaly ( $4 \mathrm{~cm}$ below the costal margin) and splenomegaly ( $5 \mathrm{~cm}$ below the costal margin) that had been found by fetal ultrasonography at week 36 of gestation. Laboratory results were as follows: White blood cell (WBC) count $16.82 \times 10^{9} / \mathrm{L}$, hemoglobin $115 \mathrm{~g} / \mathrm{L}$, platelet count $32 \times 10^{9} / \mathrm{L}$, neutrophils $1.22 \times 10^{9} / \mathrm{L}$, lymphocytes $12.72 \times 10^{9} / \mathrm{L}$, C-reactive protein (CRP) $14.8 \mathrm{mg} / \mathrm{L}$, procalcitonin (PCT) $0.75 \mu \mathrm{g} / \mathrm{L}$. A panel for immunoglobulin M(IgM) for rubella, cytomegalovirus (CMV), toxoplasma, and herpes simplex virus (HSV) was negative, as was a panel for sexually transmitted diseases. Polymerase chain reaction DNA testing for Epstein-Barr virus, CMV, and tuberculosis was negative. He was treated for bacterial infection with imipenem, erythrocyte transfusion, and intravenous immunoglobin at $1 \mathrm{~g} / \mathrm{kg}$. Over the next 5 days, his condition did not improved. Bone marrow puncture was performed and hemophagocytosis (Figure 1) was found in the bone marrow smear. Later full lab results are shown in Table 1, defective NK/CTL degranulation was detected (Figure 2). The diagnosis of HLH was eventually made and it was assumed that HLH might begin to manifest at least as early as 36 weeks' gestation. Methylprednisolone $2 \mathrm{mg} / \mathrm{kg}$ was administered and genetic tests for FHL were carried out, owing to the extremely early onset and defective NK/CTL degranulation. His fever was under control for about a week and his complete blood count showed improvement, but his condition worsened after his parents refused further intensive chemotherapy because of the genetic test showed a heterozygous mutations of UNC13D: c.2448-13 $\mathrm{G}>\mathrm{A}$ in exon26 and c.1055+1 G>A in exon12 and he died on day 27, 11 July 2014, from disease progression.

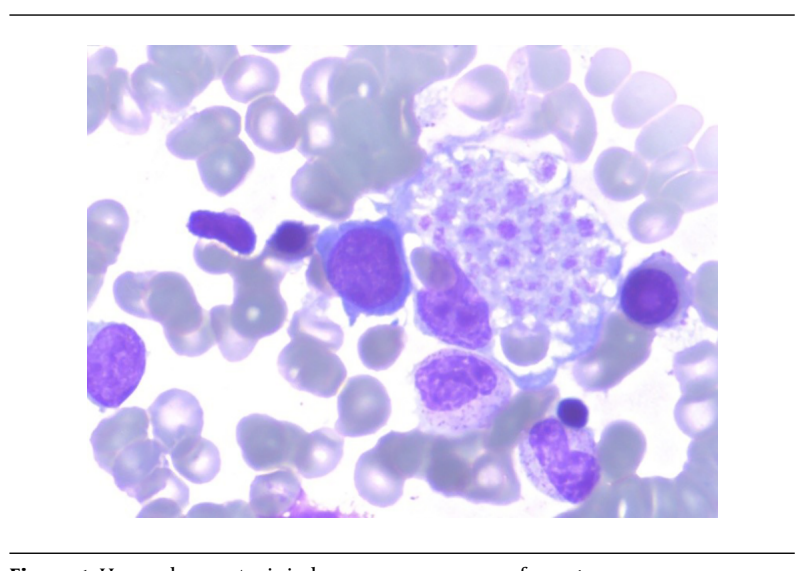

Figure 1. Hemophagocytosis in bone marrow smear of case 1

Our case 2 was a two-month-old girl and the second child of the same couple above. She was treated with antibiotics at local hospital, and her lab results demonstrated pancytopenia, elevated serum ferritin and decreased NK 


\begin{tabular}{|c|c|c|}
\hline Clinical Manifestations or Lab Results & Case 1 & Case 2 \\
\hline Fever & Occasionally & Recurrent fever \\
\hline Enlarged spleen & $5 \mathrm{~cm}$ below the costal margin & $3 \mathrm{~cm}$ below the costal margin \\
\hline Enlarged liver & $4 \mathrm{~cm}$ below the costal margin & $3 \mathrm{~cm}$ below the costal margin \\
\hline Triglyceride, mmol/L & 3.05 & 1.86 \\
\hline Fibrinogen, $\mathbf{g} / \mathbf{L}$ & 1.35 & 1.6 \\
\hline Hemophagocytosis & Yes & Not detected \\
\hline Serum ferritin, $\mathrm{ng} / \mathrm{mL}$ & 3120 & 2860 \\
\hline sIL-2R, U/mL & 57420 & 56140 \\
\hline IFN- $\gamma, \mathbf{p g} / \mathbf{m L}$ & 2133 & 1762 \\
\hline IL-6, pg/mL & 51.4 & 30.4 \\
\hline IL-10, pg/mL & $>1000$ & $>1000$ \\
\hline TNF- $\alpha$, pg/mL & 113 & 128 \\
\hline CD3+CD19-, \% & 85.2 & 88.4 \\
\hline CD3+CD8+, \% & 42.5 & 65.3 \\
\hline CD3-CD16+CD56+, \% & 3.6 & 4.5 \\
\hline$\Delta$ CD107a with NK, \% & 0.08 & -1.33 \\
\hline$\Delta$ CD107a with CTL & 1.1 & 1.1 \\
\hline
\end{tabular}

cells account. When she was admitted to our hospital, physical examination revealed recurrent fever (Tmax $38.9^{\circ} \mathrm{C}$ ), and pallor, liver was palpable at $3 \mathrm{~cm}$ below rib edge and spleen palpable at $3 \mathrm{~cm}$ below rib edge. Lab results are shown in Table 1 . Panels for common virus and STD, the same as her brother, were negative. Blood culture for bacterial, fungal and mycobacterium was also negative. When the genomic DNA sequence analysis came back the same as the brother's, the parents decided to discontinue all treatment and let her be discharged against the attending physicians' advice. She died 2 months later due to disease progression.

UNC13D mutations lead to synthetic dysfunction of Munc13 - 4 protein and Munc13 - 4 protein deficiency, causes severe NK cytotoxicity defects (2). It had been shown that the median age of FHL3 onset is 3 months, and it occurs mainly in one year of age (3). However, the fetus ultrasound findings and laboratory results in case one suggested HLH during the fetal period, while case 2 had disease onset when 2 months old. We speculate reasons for this as follows: (1) Compound disruptive mutations can lead to nearly absent granule release capacity. Sieni et al. (4) found that disruptive mutations may result in defective NK cytotoxicity and early disease onset, and (2) A trigger is an essential factor in HLH; the two cases we describe may have had different infection origins. Imashuku et al. (5) analyzed 96 infants with HLH in Japan and found that enterovirus and HSV, usually occurring within 1 - 2 weeks of birth, and EBV, usually occurring at the age of 9-12 months, were associated with HLH. HSV, EBV, CMV, rubella virus, and human immunodeficiency virus had been eliminated in our cases; unfortunately enterovirus and parasites had not been tested.

The case 2 had been mistreated at least two weeks for an infectious disease. Owing to non-specific symptoms and signs, the diagnosis of HLH is very difficult. Although initial clinical presentations are highly variable, prolonged fever (nearly 100\%) and hepatosplenomegaly (30\% - 90\%) are the most frequent symptoms and signs (1). However, fever and hepatosplenomegaly are often seen in septic shock in pediatric patients, making a differential diagnosis difficult. Xu et al. (6) found that a cytokine pattern of markedly elevated levels of IFN- $\gamma$ and IL-10 with only modestly elevated IL-6 levels has high diagnostic accuracy for HLH and may be a reliable approach to differentiating HLH from infection. The corresponding cytokine profile, and that of IL-10 and IFN- $\gamma$ exceeding the upper limit and IL6 mildly elevated, was found in our patients. In terms of screening tests for FHL, FCM for perforin and NK degranulation assay are more sensitive than the NK cytotoxicity function test. Perforin and CD107a tests can quickly and accurately identify genetic HLH patients (7). Most recently, 
A

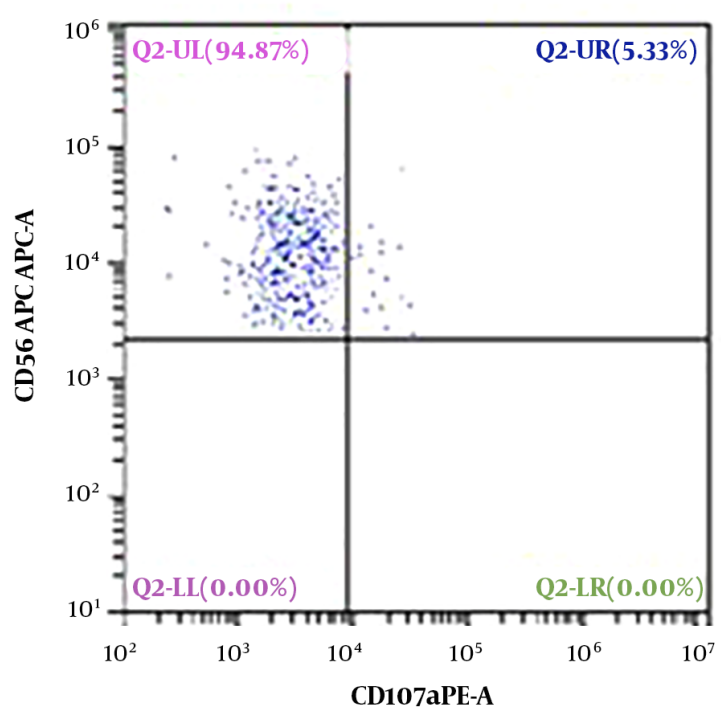

B



Prior Stimulation
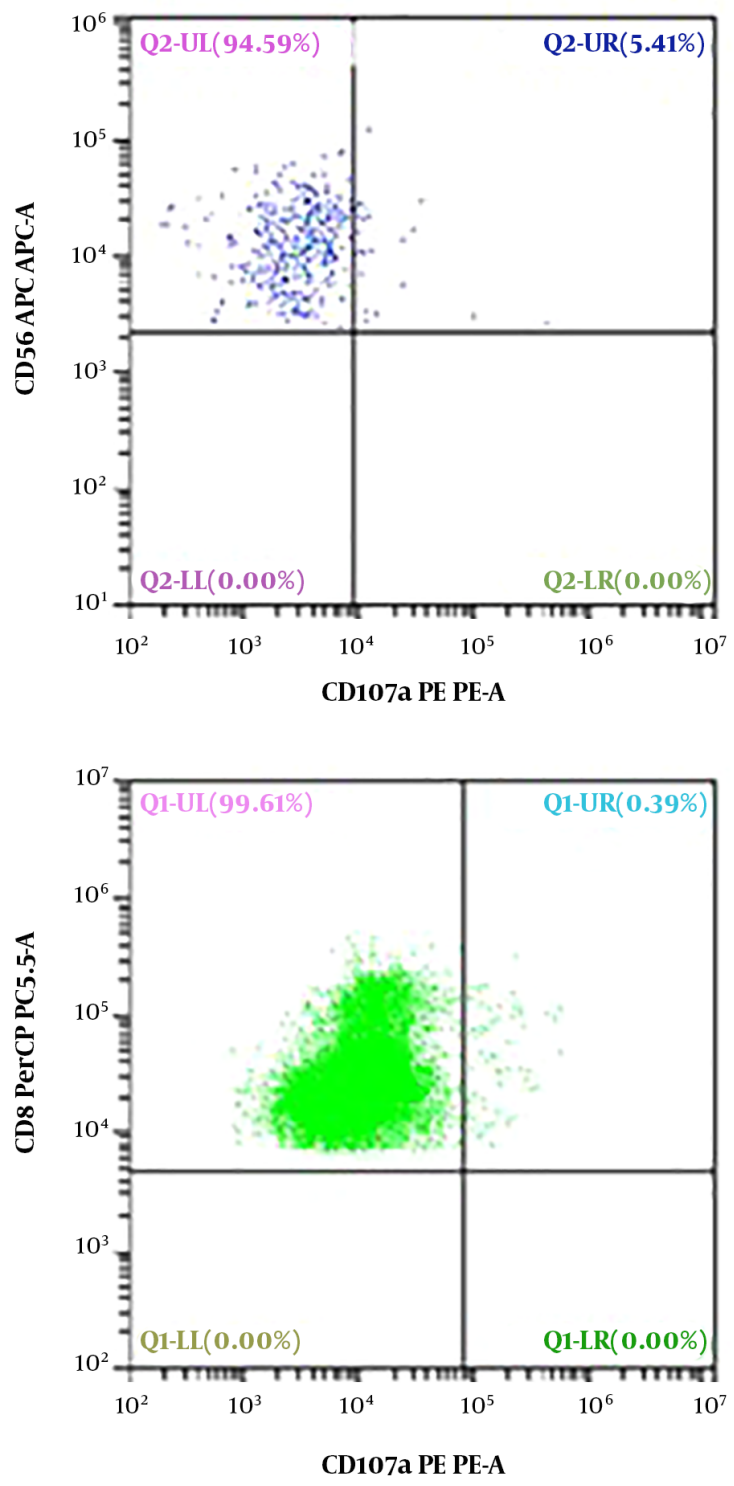

Prior Stimulation

Figure 2. Severely decreased CD107a in NK and CTL cells of case 1. A, the expression of CD107a in NK cells is 5.33\% prior to stimulation and 5.41\% post stimulation, thus CD107a is $0.08 \%$; B, CD107a mean channel fluorescence (MCF) with CTL cells was 1.1 (normal > 2.8), which is the ratio of CD107a MCF before to CD107a MCF after K562 stimulation

a CD57+CTL degranulation assay and munc13 - 4 protein expression assay had been developed to identify FHL3 patients with higher sensitivity and specificity $(2,8)$, and can be used to differentiate subtypes of UNC13D mutations. In both cases treatment was interrupted and they died short after the genetic diagnosis was established, which means FHL3 is still a rapidly progressive fatal blood disorder and needs hematopoietic stem cell transplantation with either unrelated well-matched donor or haploidentical donor (9).

In summary, despite the same compound of heterozygous UNC13D mutations, FHL3 disease onset could differ due to the different potential pathogenic triggers. Perforin and CD107a tests are effective and less time-consuming for screening FHL, so we suggest they should be carried out routinely. NGS can detect the causal genetic mutations and 
is useful for genetic counseling.

\section{Footnotes}

Conflict of Interests: None declared.

Funding/Support: This work was supported by Science and Technology Planning Project of Guangdong province, China (2017A020215126).

\section{References}

1. Meeths M, Horne A, Sabel M, Bryceson YT, Henter JI. Incidence and clinical presentation of primary hemophagocytic lymphohistiocytosis in Sweden. Pediatr Blood Cancer. 2015;62(2):346-52. doi: 10.1002/pbc.25308. [PubMed: 25382070].

2. Shibata H, Yasumi T, Shimodera S, Hiejima E, Izawa K, Kawai T, et al. Human CTL-based functional analysis shows the reliability of a munc13-4 protein expression assay for FHL3 diagnosis. Blood. 2018;131(18):201625. doi: 10.1182/blood-2017-10-812503. [PubMed: 29549174].

3. Feldmann J, Callebaut I, Raposo G, Certain S, Bacq D, Dumont C, et al. Munc13-4 is essential for cytolytic granules fusion and is mutated in a form of familial hemophagocytic lymphohistiocytosis (FHL3). Cell. 2003;115(4):461-73. [PubMed: 14622600].
4. Sieni E, Cetica V, Santoro A, Beutel K, Mastrodicasa E, Meeths M, et al. Genotype-phenotype study of familial haemophagocytic lymphohistiocytosis type 3. J Med Genet. 2011;48(5):343-52. doi 10.1136/jmg.2010.085456. [PubMed: 21248318]. [PubMed Central: PMC4115201].

5. Imashuku S, Ueda I, Teramura T, Mori K, Morimoto A, Sako M, et al Occurrence of haemophagocytic lymphohistiocytosis at less than 1 year of age: Analysis of 96 patients. Eur J Pediatr. 2005;164(5):315-9. doi: 10.1007/s00431-005-1636-9. [PubMed: 15731905].

6. Xu XJ, Tang YM, Song H, Yang SL, Xu WQ, Zhao N, et al. Diagnostic accuracy of a specific cytokine pattern in hemophagocytic lymphohistiocytosis in children. J Pediatr. 2012;160(6):984-90 e1. doi: 10.1016/j.jpeds.2011.11.046. [PubMed: 22226576].

7. Rubin TS, Zhang K, Gifford C, Lane A, Choo S, Bleesing JJ, et al. Perforin and CD107a testing is superior to NK cell function testing for screening patients for genetic HLH. Blood. 2017;129(22):2993-9. doi: 10.1182/blood-2016-12-753830. [PubMed: 28270454]. [PubMed Central: PMC5766842].

8. Hori M, Yasumi T, Shimodera S, Shibata H, Hiejima E, Oda H, et al. A CD57(+) CTL degranulation assay effectively identifies familial hemophagocytic lymphohistiocytosis type 3 patients. J Clin Immunol. 2017;37(1):92-9. doi: 10.1007/s10875-016-0357-3. [PubMed: 27896523].

9. Messina C, Zecca M, Fagioli F, Rovelli A, Giardino S, Merli P, et al. Outcomes of children with hemophagocytic lymphohistiocytosis given allogeneic hematopoietic stem cell transplantation in Italy. Biol Blood Marrow Transplant. 2018;24(6):1223-31. doi: 10.1016/j.bbmt.2018.01.022. [PubMed: 29410181]. 